ReVISTA InTERnacional de Sociología (RIS)

Vol.67, N 2, Mayo-Agosto, 347-371, 2009

ISSN: $0034-9712$

DOI: 10.3989 /ris.2006.06.08

\title{
ACTIVIDAD SOCIOCOGNITIVA Y CONTEXTOS POSTFORDISTAS
}

\section{SOCIOCOGNITIVE ACTIVITY AND POSTFORDIST CONTEXTS}

\author{
Francisco Quintana Castelló \\ Universidad de Barcelona. España \\ fqcastello@yahoo.es
}

\begin{abstract}
RESUMEN
En el capitalismo contemporáneo la actividad económica productiva se metamorfosea para configurar un paisaje híbrido, mezcla de modalidades avanzadas basadas en la microelectrónica y el lenguaje, postfordistas, y componentes tecno-organizativos y contractuales, algunos de los cuales evocan el factory system manchesteriano. Estos cambios comportan una profunda transformación del trabajo y de los sistemas técnicos y organizativos, perfectamente diferenciados de los que aparecen definidos por las tesis "endógenas" (versión smithiana y ley de Kaldor). En los nuevos prototipos, que recurren a sistemas automáticos y telemáticos y a formas de productivas relacionales, cognitivas y lingüísticas, el conocimiento se erige en fuerza productiva de primer orden. Un conocimiento, sin embargo, que se nutre sobre todo de un entorno innovador y del saber social sistémico y no sistémico. No obstante, esto conlleva también el surgimiento de nuevas problemáticas entre las que destaca el carácter dicotómico de algunos de los procesos generadores de conocimiento valorizante en un contexto global determinado por la lógica del activo.
\end{abstract}

\section{Palabras Clave Adicionales}

Conocimiento valorizante, Endógeno, Tecno-organizativo, Smithiana.

\begin{abstract}
In contemporary capitalism, productive economical activity changes into configuring a hybrid landscape formed by a mixture of advanced modalities based on microelectronics and language, which are postfordist, and techno-organizational and contractual components evoking, some of them, the manchesterian "factory system". These changes involve a deep transformation of work and technic systems and organizations distinctly differents from those which appear defined by "endogenous" thesis (Smith's version and Kaldor law). In new prototypes, turning to automatic and telecommunication systems as well as to a relational, cognitive and linguistic productive forms, knowledge raises as capital productive force. Nevertheless, most of all, this knowledge is fed by an innovative environment and a systemic and not systemic knowledge. However, these changes involve the emergence of new problems, being to emphasize the dychotomic character of those processes which generate valuating knowledge in a global context determined by asset logic.
\end{abstract}

\section{Additional KeYWords}

Endogenous, Smithian, Techno-organizational, Valuating Knowledge. 


\section{ESCENARIO NEO/POSTFORDISTA ${ }^{1}$}

Un aspecto insoslayable de las formaciones sociales contemporáneas es que una porción importante de las transformaciones acontecidas concierne al trabajo y a su relación con los procesos tecno-organizativos; repercuten en la interrelación entre trabajo, sistemas técnicos y sistemas organizativos. Ahora bien, en cuanto a las interpretaciones surgidas sobre esta cuestión, algo que hay que destacar es que la idea que transmiten algunas de las investigaciones más innovadoras, particularmente las provinentes de los teóricos del "ciclo de producción inmaterial", es que las nuevas tecnologías (redes telemáticas, automática industrial, software aplicado a sistemas embedded,...) y el new management están ampliamente difundidos, sobre todo en las regiones industrializadas, hasta el punto de considerar que aquellos son algunos de sus rasgos más representativos.

No obstante, si examinamos estos cambios con mayor detenimiento veremos que, a diferencia de lo apuntado por aquellas lecturas, no han conducido precisamente a un escenario caracterizado por la omnipresencia high tech y del trabajo cognitivo (telemático, en redes, etc.). Lo que se percibe es algo distinto, lejos de la uniformidad high tech; paisaje heterogéneo en el que se mezclan de manera no siempre sistemática, en los distintos ámbitos (regiones, sectores, segmentos, puestos de trabajo, etc.), una variada gama de modalidades tecno-organizativas y contractuales que van desde las que al menos en sus componentes básico ya se daban en los albores del capitalismo productivo, con anterioridad al factory system, hasta las que se basan en las Tecnologías de la Información y la Comunicación (TIC) y prototipos organizativos dependientes de la "gestión de intangibles".

Escenario, con un sinfín de modalidades, en el que se vislumbran grosso modo dos polaridades, esto es: un polo, que correspondería a las regiones periféricas de la "socioeconomía mundo", donde predominan el neofordismo y el neotaylorismo, bajo en intensidad tecnológica y organizativa y con unas condiciones sociolaborales extremadamente precarias (Zonas de Procesamiento para las Exportaciones, maquilas); y otro, postfordista (y postaylorista), intenso en TIC y que se apoya en la figura del knowledge worker. Además, uno de los rasgos más destacados de esta geometría variable es que en el centro encontremos la periferia (neofordismo y workpoor en EEUU y Unión Europea), y en la periferia el centro (recurso a las TIC en la India) (Brenner\&Theodore,

\footnotetext{
${ }^{1}$ Queremos señalar que en este artículo se hace un uso genérico de los términos neofordismo (individualización y rutinización de tareas y puestos; fragmentación de tiempos y movimientos; recurso parcial a las TIC, ) y postfordismo (TIC; trabajo sociocognitivo; cooperación productiva, etc.) para designar los dos grandes enfoques que en líneas generales existen en lo referente a las propuestas analíticas acerca de los procesos tecno-organizativos contemporáneos y que, al igual que su precedente -"modelo fordista"- basado en el pacto social capital-trabajo, en un fordismo, denotan el influjo del "regulacionismo", pero, sobre todo, poseen una insoslayable dimensión política. Para un examen más específico de estos conceptos ver Lipietz (1990, 1996); Marazzi $(2003,2004)$; Zanini\&Fadini (2001) y Bologna (2006).
} 
2002; Arrighi y Beverly, 2001; Harvey, 2004, 2007; Klein, 2001; Quintana, 2002, 2006; Wallerstein, 2007).

Hay que decir que España ocupa, en este periodo histórico, una posición intermedia. Efectivamente, con arreglo a los datos del Instituto Nacional de Estadística (INE), en el periodo 2000-2004, solamente 51.319 empresas, el $29,7 \%$ del total, fueron tecnológicamente innovadoras, entendiendo por tales las que realizaron actividades en este ámbito, evaluadas según determinados indicadores: I+D (interno o externo); adquisición de maquinaria, equipos y software; adquisición de otros conocimientos (patentes, licencias,...); formación, etc. Por otro lado, el Summary Innovation Index (SII) elaborado por la Comisión Europea (gráfico 1) indica que a España le corresponde, dentro del conjunto de los 34 países más industrializados del la OCDE, con 0,30 puntos, la posición número $20^{2}$.

Así pues, si recurrimos a las categorías que tienen su origen en las teorías de la regulación (Aglietta, Boyer, Coriat, Lipietz), consideradas uno de los aportes más fructíferos en este campo, diremos que lo que caracteriza al actual periodo es la existencia de un cóctel de neofordismo (taylorismo renovado, fordismo sin las garantías del "pacto fordista-keynesiano", etc.), y postfordismo (postaylorismo, recurso a las TIC y a formas organizativas que descansan en el saber de la ciencia y de la técnica, así como en el saber social en general,). Se trataría, por tanto, de un escenario híbrido.

Desde otro ángulo, una de las características de esta situación sin precedentes, que dota a los procesos productivos de una particular singularidad, es que la lógica de la valorización se expande y penetra la generalidad de los objetos, relaciones e instituciones (estamentos públicos, servicios, recursos básicos, etc) de la mano del productivismo y de la financiarización de la economía, para invadir las distintas fases del ciclo económico: producción, circulación (pequeña y gran circulación), reproducción, uno de cuyos efectos ha sido que se difumina la clásica distinción marxiana entre actividades productivas e improductivas en aras de una nueva productividad general -sinérgica, "integral"- de lo social y del territorio ${ }^{3}$.

\footnotetext{
${ }^{2}$ El indicador Summary Innovation Index (SII), que ha sufrido alguna modificación parcial después del 2004, puesto que de él se han excluido entre otras cosas el porcentaje de capital riesgo invertido en alta tecnología, engloba indicadores sobre sistema educativo en la educación secundaria, inversión en I+D en áreas de intensidad alta y media-alta, gasto en I+D universitario aportado por capital privado, protección de derechos de propiedad intelectual (patentes triádicas, marcas comerciales y diseños comunitarios), etc.

${ }^{3}$ J. C. Delaunay (2001, pp. 115 ss.) realiza una importante contribución a la teoría del valor en los servicios a partir, en primer lugar, del concepto de "bien inmaterial" susceptible de transacción de Say y, por otra parte, de la teoría de los "bienes internos" del economista ruso Heinrich Storch. La relevancia de las indagaciones de Storch estriba en que, al analizar aquel tipo de prácticas y, concretamente, la actividad de consulta (prescripción médica,...), establece una distinción entre productos categorizados como no intercambiables (no mercantilizables), y servicios efectuados, cuyo resultado son productos, que sí son intercambiables, susceptibles de transacción, y, también, valorizantes. La aportación de Delaunay tiene, además, el interés de que permite incluir entre las actividades que en determinados contextos valorizan algunas que desde la ortodoxia crítica no se consideraban como tales (logística, consultoría, sanidad, enseñanza, etc.), lo que
} 
Gráfico 1.

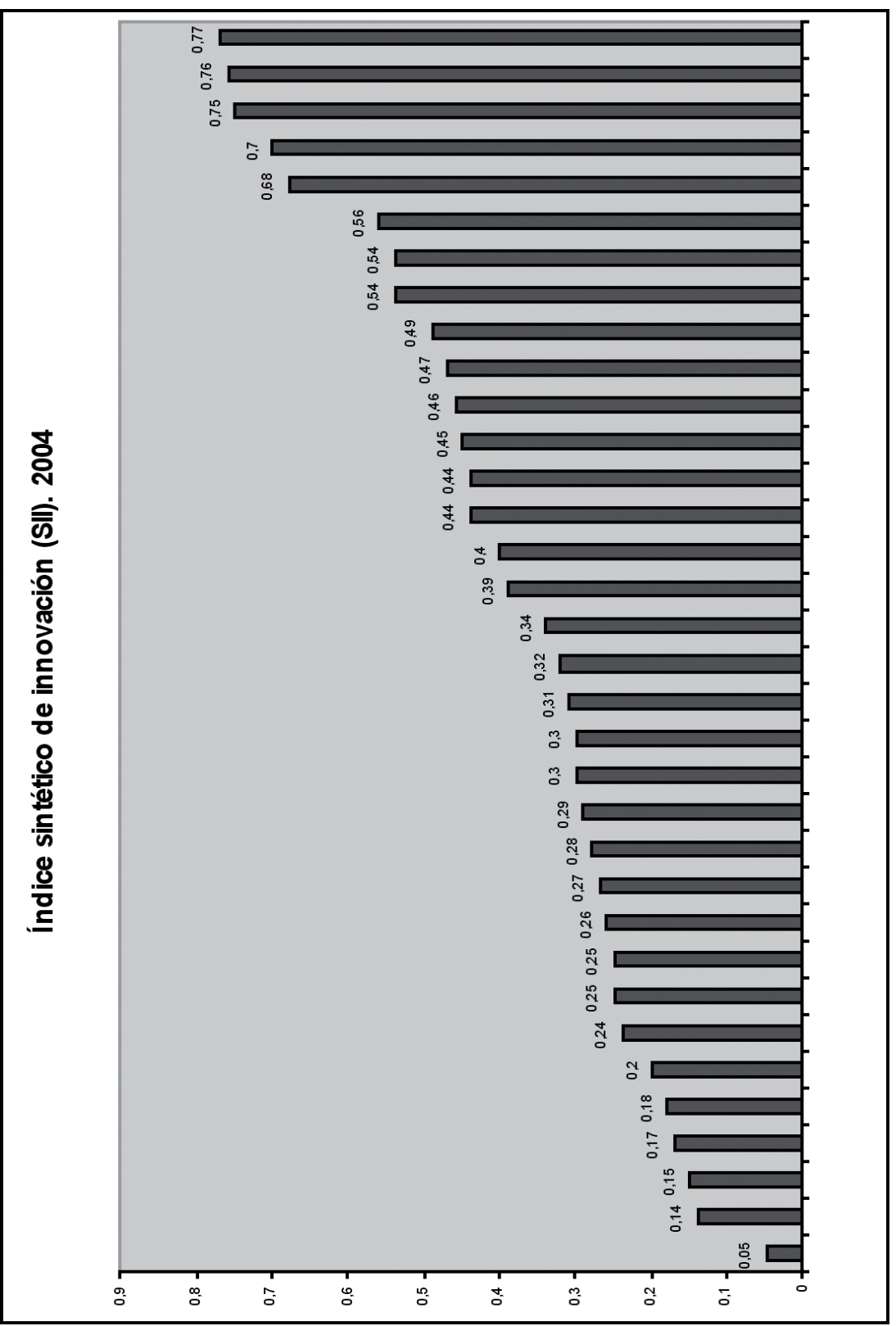

Fuente: Innovation Scoreboard. European Commission (2004). 
En cualquier caso, es en este marco -neo/postfordista- donde transcurre la metamorfosis del trabajo y de los procesos tecno-organizativos; mutación de los procesos resultantes de la interacción entre el trabajo, la tecnología o los sistemas técnicos y las modalidades organizativas en tanto que aspectos indisociables integrantes de un único, y complejo, proceso global para el incremento del value added.

\section{ENDÓGENOS Y CRÍTICOS}

Iniciaremos, pues, este periplo sobre la metamorfosis del trabajo y tecno-organizativa prestando especial atención a la actividad relacional y cognitiva, así como a la vertiente postfordista del proceso económico. Previamente, sin embargo, definiremos una posición -lugar de la enunciación- analítica e interpretativa, acerca de cómo se concibe el crecimiento socioeconómico y, como una parte de él, la innovación tecnológica y organizativa; punto de partida en el que se cuestionan las tesis "endógenas" al considerar que las estructuras productivas conforman procesos de naturaleza social y territorial que sobrepasan la esfera de lo que jurídica y formalmente representan los confines de las corporaciones y de lo económico.

Así, de entrada nos referiremos a las dos grandes propuestas que con múltiples ramificaciones hay sobre este tema, esto es:

a) Perspectiva "endógena" (Kaldor, Friedman, Lucas, Romer, Young), anclada en la tradición neoclásica, y caracterizada por situar en el centro, como motor de la innovación y el crecimiento económico, al capital y a sus actores-jerarcas.

b) Por otro lado, tenemos una panoplia de autores (Bologna, Chesnais, Corsani, Lazzarato, Marazzi, Moulier, Vercellone) que con distintos matices y diferencias comparten su rechazo de las tesis del crecimiento "endógeno", catalogadas de asociales y abstractas, frente a las que plantean abordar de otro modo, desde la indexicalidad y pluridimensionalidad, tanto los elementos y relaciones internos tecnoorganizativos y de trabajo como el contexto.

A este respecto, hay que decir, en primer lugar, que la corriente "endógena" remite a la Riqueza de las naciones de Adam Smith y a una concepción según la cual la productividad y el crecimiento económico dependen, por una parte, de la división técnica del trabajo y de la especialización y, por otra, de la extensión de los mercados. Una formulación posterior de este enfoque es la que ha tomado como referencia la ley Kaldor-Verdoon.

posibilita, al propio tiempo, afianzar el concepto de itinerario socioeconómico "integral" en los términos que en este texto se proponen. 
Con arreglo a dicha ley, existe un vínculo favorable entre el crecimiento de la producción industrial y el crecimiento de la productividad del trabajo. Kaldor alude, también, a la vinculación entre división del trabajo, especialización obrera y crecimiento económico, y propone como medidas concretas invertir en equipos, incrementar la división técnica y la especialización en el trabajo y, finalmente, ahondar en la especialización de las industrias. Esto implica que el desarrollo va a depender de los resultados obtenidos en el aprendizaje de la fuerza de trabajo y de la inversión en capital fijo. De este modo, a las propuestas clásicas se suman, aunque con algunas discrepancias, las nuevas ideas del management partidario de la interiorización del progreso técnico y del crecimiento con arreglo a la pautas del learning-by-doing. Sin embargo, este punto de vista, en el que la contribución de la fuerza de trabajo y el entorno sociocultural no desempeñan más que un papel subalterno, pasivo, supeditado al par pensantes/ejecutantes, ha mostrado con la crisis del modelo "fordista" o "fordista keynesiano" sus propias limitaciones.

A pesar de ello, más recientemente encontramos de nuevo, con un talante continuista respecto de los neoclásicos, aunque con retoques, la propuesta "endógena". Una de las consecuencias de su aplicación ha sido, como han señalado Herrera y Vercellone (2003), la segmentación dual del mercado de trabajo, así como la distinción entre el "componente intelectual de la fuerza de trabajo" (capital humano reproductible) y el resto de la fuerza de trabajo (trabajo no cualificado). Sin embargo, esta propuesta no representa ninguna novedad, ya que en realidad se trata de un remake de la dinámica de "descalificación-sobrecualificación" que ha seguido los pasos de la Escuela de Chicago (Friedman, Becker, Lucas).

Con todo, aun teniendo en cuenta el poderoso influjo ejercido por el "endogenismo", como un componente de lo que en teoría económica se designa como "corriente principal", tal enfoque no ha dejado de ser fuertemente cuestionado. Veamos someramente algunas de las críticas. Primeramente nos referiremos a las efectuadas por Herrera y Vercellone (2003, pp. 48-49). Lo que destacan estos autores es que, por lo que se refiere al papel de la fuerza de trabajo y del conocimiento productivo, no hay diferencias cualitativas notables entre las distintas variantes "endógenas". En todos los casos permanece sin apenas variación la impronta conceptual de Adam Smith: división social y técnica del trabajo; la innovación es interna a la producción y extraña al entorno; asocialidad; papel central atribuido a los actores jerarcas; dinámica dicotómica que relega a la fuerza de trabajo descualificada, etc. Por contra, en lo que hacen hincapié Herrera y Vercellone al referirse a las modalidades tecno-organizativas y de producción actuales es en que el "verdadero motor" de la producción de conocimiento en tanto que fuerza productiva no es, aunque dependa de él, la consecución del beneficio, sino que es el conjunto de dinámicas surgidas a partir de lo que calificamos como "dimensión cooperativa" de lo social; un rasgo que sobrepasa la propiedad corporativa; factor que depende en lo esencial del bagaje competencial y sociocultural de la población, así como del saber no mercantilizado. Podríamos decir, por tanto, que aquello que subyace en la concepción "endógena" es un postulado, a priori metafísico, según el cual habría un corte, una separación, entre procesos económicos y procesos sociales, lo cual nos conduce al 
absurdo de que un tipo de actividad, como es el caso de la economía, básicamente de carácter social, se realice entre actores que no interactúan entre sí. En este caso resulta perfectamente aplicable el comentario de Bourdieu (2000) en el que se dice que la ciencia llamada Economía descansa en una "abstracción originaria" al eludir la naturaleza (base ontológica) social de los procesos económicos.

Por su parte, Castells y Hall (1994, p. 30), ponen también el acento en el peso específico del contexto al definir el concepto de "medio innovador" entendido como: "[...] el sistema de estructuras sociales, institucionales, organizativas, económicas y territoriales que crean las condiciones para una generación continua de sinergias y su inversión en un proceso de producción que se origina a partir de esta capacidad sinérgica, tanto para las unidades de producción que son parte de ese medio innovador como para el medio en su conjunto". Algo que corrobora la importancia que reviste el hacer hincapié en los elementos contextuales es que instituciones tan convencionales como el World Competitiveness Center, adscrito al International Institute for Management Development (IMD) de Lausanne incluye entre los denominados "factores de competitividad" a la familia de indicadores "societal framework", que engloba el factor "cohesión social".

Por fin, desde un óptica diferenciada de las anteriores (Gaudemar, 1981; Deleuze\&Guattari 1988; Guattari, 1996; Ilardi 1999; Negri, 1979 y 1992; Virno, 2001; Berardi, 2001) se constata que tras la crisis y reconversión el nexo capitalista ha mutado en "ciclo de producción integral". Un aspecto importante de esta mutación -promovida mediante las TIC y las formas de organización cooperantes- estriba en la relevancia atribuida a las dimensiones socioligüísticas, comunicativas y del comportamiento, a diferencia de lo que ocurría con el fordismo y el taylorismo que, al decir de Virno (2001 $a, b)$, discurría en silencio. Lo que sucede es que, en el interior y en el exterior de la empresa, los flujos de sociabilidad y relacionales emergen como filón privilegiado para la producción desbordando con creces los límites fijados por las coordenadas smithianas y neosmithianas.

A este respecto, Gorz (2003) y Marazzi (2003) han subrayado la importancia en la vertiente postfordista de esa nueva forma de "capital fijo", distinta al "capital fijo" tradicional, compuesta por ingredientes inmateriales (relaciones, formas de vida, expresiones lingüísticas, lenguajes formales, informaciones, etc.), no cuantificables, que se gestan en lo social, en el puzle de los saberes sociales. Conjunto de conocimientos, parte de ellos, finalmente codificados por la matriz informacional; informaciones que se prefiguran como principio activo fundamental. Esto quiere decir que con los nuevos prototipos productivos lo que cuenta no es o no es únicamente el conocimiento de la técnica y de la ciencia, sino también ese otro tipo de conocimientos que germinan en el caldo de cultivo de los saberes relacionales y comunicativos.

Vistas así las cosas, acto seguido profundizaremos en esta perspectiva que conecta metamorfosis del trabajo y flujos de sociabilidad y relacionalidad. 


\section{TRABAJO, ACTIVIDAD SOCIOCOGNITIVA Y GRUPO}

Ciertamente, tal y como se desprende de la crítica a la postura "endógena", el cambio cualitativo en el ámbito del trabajo y del valor respecto de las formas smithiana ("fábrica de agujas") y fordista (y taylorista) ha originado presumiblemente determinadas configuraciones tecno-organizativas basadas en última instancia en la expropiación de la productividad generada por lo social y por el entorno. Sin embargo, esto no quiere decir que todos los comportamientos y relaciones -las prácticas sociocognitivas- sean valorizantes, pero sí que, en la actualidad, ante todo en las secuencias postfordistas, intensas en técnica y organización, la producción y el plusvalor se alimentan de la productividad de un sinfín de elementos que surgen en las relaciones sociales y en la intersujetividad; elementos que de un modo directo o indirecto, como requisitos o como prerrequisitos, colaboran con el soporte informacional y telemático al incremento del valor del capital.

En este sentido, los "distritos marshallianos" del s. XIX -seda de Lyon, cuchillería y aceros especiales de Solingen, Remscheid y Sheffield, productos de algodón procedentes de Filadelfia y Pawtucket- así como los "distritos industriales" formados por redes de pequeñas y medianas empresas innovadoras y muy competitivas, que tienen sus raíces en las culturas locales y en los saberes artesanales (Piore\&Sabel, 1990; Amatori, 1997; Instituto per la Promozione Industriale, 2003), caso de Italia, inspiradores de las actuales cluster economies, todos ellos, en conjunto, bien pueden ser considerados uno de los más claros antecedentes de esta visión indexical del desarrollo socioeconómico-surgida tras la reconversión de los 70- que preconiza la captura de los flujos relacionales: comportamientos, subjetividades y saberes. Concepción novedosa que inscribe los flujos de saber social y de subjetividad y, como un aspecto de estos, los "sistemas de innovación", en el seno de la propia división tecno-organizativa del trabajo y de la producción.

A este respecto, en lo concerniente a los recursos del entorno, el papel de las instituciones y de los grupos de poder ha sido crucial. Veamos tres casos relevantes. En primer lugar, esto es lo que ha sucedido, por ejemplo en España, con la reformulación del sistema educativo que debe evolucionar en sus diversas ramas (EGB, ESO, FP, Ciclos Formativos, etc.) acorde con las necesidades formativas de la fuerza de trabajo. Aquí el primer paso se dio con la aprobación de la Ley General de Educación Villar Palasí de 1970. En segundo lugar, la implantación de los modelos I+D e I+D+i ilustran la subordinación de la investigación a los procesos socioeconómicos; sobre esto resultan sumamente clarificadoras las primeras directrices sobre I+D elaboradas, en 1968, por el Ministére du Développment Industriel et Scientifique francés, en las que se afirmaba que era imprescindible que hubiera "utilización de los resultados de investigación para llegar a la explotación de nuevos productos, dispositivos, sistemas y procedimientos, 0 para mejorar los ya existentes. El desarrollo consiste en pasar del modelo de laboratorio, que muestra la validez de los principios elegidos para un nuevo procedimiento industrial, al prototipo industrial digno de ser reproducido para la venta". Por último, estaría la 
propagación de determinado enfoque de la cultura cívica generador de cohesión social, que, con el apoyo de cierta normativa, puede contribuir al modelado de cuerpos y subjetividades, caldo de cultivo y prerrequisito de productividad de lo social. Cultura cívica, por cierto, que ha sido la pieza fundamental del auge del competitive corporatism, modelo que vimos funcionar en el pacto "Moncloa 77" y clave del éxito del celtic tiger (Nolan, O'Connell\&Whelan, 2000; Kirby, 2002).

Por otra parte, si nos circunscribimos a los aspectos más concretos del trabajo, podremos observar que si el fordismo estuvo caracterizado por el trabajo individualizado, repetitivo, en cambio el postfordismo potencia la actividad productiva grupal y la comunicación (Vincent, 1993; Cillario, 1996; Zarifian, 1998; Virno, 2001 a, b; Marazzi, 2002 y 2003; Gorz, 2003). Veltz (2000: 16-18) afirma, al respecto, que es preciso distinguir entre el "individuo genérico" del mundo industrial clásico, aún vigente, capaz de llevar a cabo tareas programadas, y el individuo cooperativo de los sistemas de trabajo avanzados. Aquel individuo es parte de un colectivo en el que las operaciones se desarrollan por adición, están seriadas, y donde la comunicación interpersonal tiene un papel marginal o parásito. Y esto sucede a pesar de que se sabe que hay un reserva de inteligencia y de saber involucrados, dispuestos para resolver las "disfunciones" que surgen en el "sistema programado". Sin embargo, a pesar de todo, en muchos casos la comunicación y la autonomía no dejan de recortarse hasta quedar anuladas. No obstante, en un número cada vez mayor de contextos, esto no funciona. Y ello se debe a que las tareas y los procesos están en función preferentemente no de la intensidad del trabajo, de la carga de trabajo programada, sino de las relaciones intergrupales y entre los individuos en cada tramo y en cada situación; además, en ocasiones estos procesos se ven distorsionados por factores no programados, aleatorios. En este sentido, puede decirse que es la organización concebida en términos de calidad de la cooperación y de las "interfaces" entre los actores y la cadena de producción la piedra angular de estos procesos. En esta modalidad, basada en los conceptos de performatividad y eficacia, lo que prima es el grupo y no el individuo.

Aquí destacan, por tanto, la aplicación de formatos cooperantes (grupos de trabajo, flexibilidad, autonomía), preferentemente en áreas industrializadas, en diversos puestos y sectores, a saber: química, farmacia, automóvil, enseñanza, sanidad, servicios a las empresas (consultoría, asesoría fiscal); inmobiliario, etc., que recurren a las TIC. Asimismo, ciertos datos relativos a la organización del trabajo en la Unión Europea ilustran de un modo patente el influjo de aquellas concepciones, lo que tiene su plasmación en la proliferación de elementos y sistemas diferenciados e incluso opuestos a las modalidades precedentes, fordista y taylorista. En efecto, un estudio exhaustivo sobre las condiciones de trabajo y tecno-organizativas de asalariados de la Unión Europea, patrocinado por la Fundación Dublín y realizado por Daubas, Letourneux y Thébaud-Mony (2002) ${ }^{4}$, sostiene que: autoevalúan la calidad del trabajo el $69 \%$ de las

\footnotetext{
${ }^{4}$ El estudio ha excluido aquellos que trabajan menos de 10 horas diarias y la agricultura.
} 


\section{Gráfico 2.}

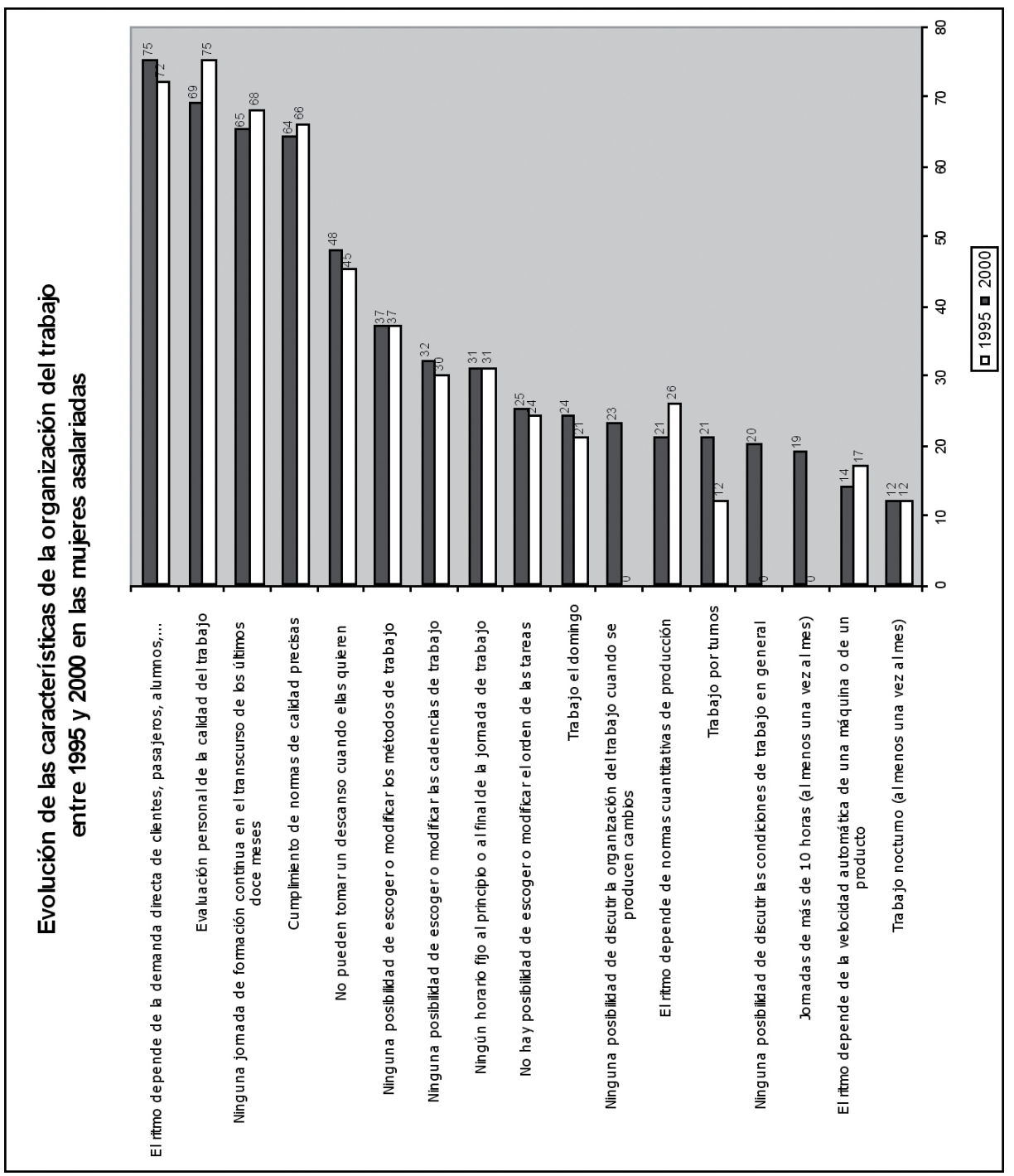

Fuente: Organisation du travail et santé dans I ' Union Européenne (2002). 


\section{Gráfico 3.}

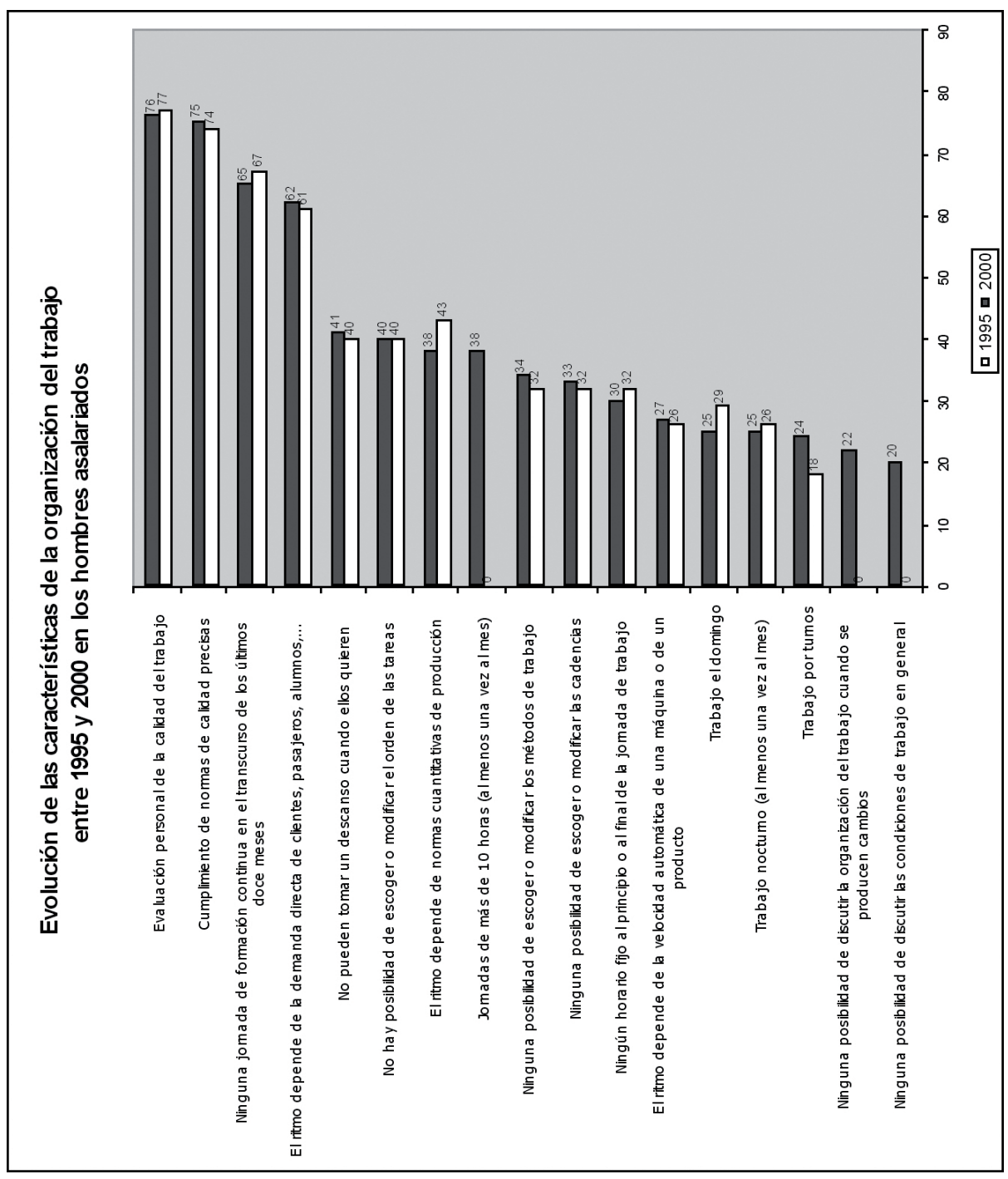

Fuente: Organisation du travail et santé dans I ' Union Européenne (2002). 
mujeres y el $76 \%$ de los hombres; pueden escoger o modificar los ritmos de trabajo el $68 \%$ de las mujeres y el $67 \%$ de los hombres; pueden elegir o modificar los métodos de trabajo el $69 \%$ de las mujeres y el $66 \%$ de los hombres; pueden discutir los cambios que se producen en la organización del trabajo el $77 \%$ de las mujeres y el $78 \%$ de los hombres; pueden discutir las condiciones generales del trabajo el $80 \%$ de las mujeres y el $80 \%$ de los hombres. Para tener una visión más detallada de estos cambios, veamos un par de gráficos (gráficos 2 y 3 ), pertenecientes al mencionado estudio, en el que se incluyen indicadores que permiten trazar un perfil específico de aspectos básicos de la organización del trabajo en la UE y de su evolución entre 1995 y 2000.

No obstante, hay que puntualizar que se trata obviamente de cifras promedio. Así, según el informe Employment at Glance 2002 de la OCDE (Navarro, 2006: 106 y ss.), en España, semiperiferia capitalista, el $55 \%$ de los trabajadores en situación precaria y el $50 \%$ de los fijos manifestaban que sus condiciones de trabajo eran insatisfactorias; además un $57 \%$ de fijos y un $68 \%$ de precarios afirmaban carecer de autonomía y no poder influir en su trabajo.

En este sentido, lo ocurrido en las grandes pirámides de "subcontratación" promovidas por las corporaciones y, en particular, por el sector de la automoción, ratifica, sin duda, la heterogeneidad que presenta la geografía del escenario postfordista. A este respecto, Gorz (1998: 57-58), al referirse a Toyota, señalaba que es en la casa matriz, que agrupa al $15 \%$ del total de la fuerza de trabajo que interviene en la cadena del valor y que realiza solamente tareas de ensamblaje, el único ámbito en el que se aplica estrictamente el "ohnismo" o "método Toyota" (kaizen, círculos de calidad, Método de Órdenes Desde el Final-MODF, just in time, etc.) . El resto de las operaciones se lleva a cabo mediante un enjambre de empresas, 45.000 , que conforman una pirámide en la que dependiendo del estrato que se ocupa y de la distancia al vértice superior, donde se ubica la empresa matriz, los ingredientes postfordistas desaparecen gradualmente y son reemplazados por un combinado de taylorismo y neofordismo (disminución de la intensidad tecnológica, jerarquización rígida, metodologías no cooperantes, fraccionamiento, rutinización, inestabilidad laboral).

Por otro lado, en lo tocante a la difusión de las TIC y a la tipología de organización del trabajo postfordista, aparte del sector asalariado, hay que tener en cuenta también, dada su amplitud, el ámbito del "trabajador autónomo de segunda generación" (TASG); modalidad de self-employment que no hay que confundir con la enterprise o empresa autónoma. Esta figura, diferenciada de las formas de trabajo tradicional (artesanos, agricultores independientes), abarca un amplio espectro de actividades (proveedor cualificado de grandes empresas, logística, servicios a las personas, sector irregular o "sumergido"). Dichas actividades, como han remarcado Bologna $(1997,1998,2006)$ y Belussi (2001), están ligadas en muchos casos a las redes globales y locales, a la network economy, en los servicios y en la manufactura, y se apoyan en formas de trabajo basadas en las habilidades relacionales y de comunicación cuya herramienta primordial son las TIC. 
En síntesis, podemos afirmar que, en líneas generales, el postfordismo comporta la implantación de formatos en que la demanda de implicación sociocognitiva, el espacio/ tiempo del trabajo, sus exigencias y efectos, se prolonga más allá del espacio/tiempo presencial; los límites se estiran, saltan por encima del binomio tiempo de trabajo/ tiempo de ocio para invadir la totalidad de las "formas de vida". Desbordan la separación entre trabajo y no trabajo y se extienden por el territorio para engullir y metabolizar la relacionalidad, las expresiones ligüísticas y los valores.

Hemos pasado, por consiguiente, si recurrimos al lenguaje de Habermas, de la sociedad de la "acción instrumental" a una sociedad en que la "acción comunicativa" que circula por la red telemática se supedita a la "acción instrumental".

No obstante, hay que precisar que la introducción de estos procedimientos, como por ejemplo los grupos de trabajo (GT), no ha estado exenta de resistencias (General Motors, Volkswagen, Alstom, Ciba-Geigy). Sobre este particular, hay que decir que, estas y otras discrepancias, "problematizaciones", sobre un trasfondo de deterioro del consenso social y político, o bien del declive del modelo competitive corporatism (Rhodes, 1998), explicarían las dificultades surgidas a la hora de instaurar estas formas de organización del trabajo; aunque, también tendrían que ver con los cambios operados en las prescripciones postfordistas, sobre todo en el mundo asalariado, en virtud de lo cual los criterios cooperantes han derivado en imperativo de la cooperación; postfordismo imperativo.

Por fin, en cuanto a la génesis de estas modalidades podríamos considerar que las sinergias postfordistas no son ajenas a una hipotética conversión de las modalidades de cooperación, de los comportamientos conflictivos y antagónicos surgidas en el seno de las luchas obreras y sociales, y, en particular, de las que emergen, de manera explícita 0 dispersa, entre los 60 y los 70; captura y transmutación de unos vínculos comunicativos y de cooperación, que trastocaban el sentido del valor y del trabajo, autovalorizantes (Negri, 1992, Boltanski\&Chiapello, 2002).

\section{SABER SOCIAL Y CONOCIMIENTO VALORIZANTE}

En esta tesitura, el saber social y el conocimiento que añade valor directamente - conocimiento valorizante- se perfilan como la columna vertebral de las nuevas modalidades productivas. No obstante, hay que señalar aquí que cuando hablamos de conocimiento no nos referimos única y exclusivamente a los filones de saber operativo, conocimiento convertido en información, capaz de ser interpretado por la matriz informacional - fuzzy logic, lingüística y microelectrónica - aludimos también a otro tipo de saber depositado en lo social. Saber social, sedimentado o manifiesto, que vislumbramos en las relaciones sociales; expresiones, códigos, costumbres, formas de comunicación, esquemas de interpretación, valores, etc., que representan también para las corporaciones un valioso filón de materia prima que hay que transformar y reconducir hacia la esfera de lo funcional. 
Con todo, no hay que olvidar que en los comienzos del capitalismo productivo, organización social y económica que se gesta en el siglo XVIII, el saber ya tenía un papel relevante. Entonces, cabe preguntarse ¿cuál es la diferencia entre el postfordismo y las modalidades anteriores? Que duda cabe, hay varias cosas que distinguen el ligamen que se establece, por ejemplo, entre ciencia aplicada y sistema productivo. En este sentido, en la actualidad este conocimiento ha sido internalizado por el proceso de trabajo y de "producción integral", no es algo que discurra en paralelo y que periódicamente tienda puentes de colaboración como ocurría en épocas anteriores. En el postfordismo, el desarrollo del saber científico y técnico es parte de los procesos de reproducción ampliada de capital. Los modelos I+D o I+D+i son una muestra palpable de la absorción de dichos saberes por el circuito de la mercancía.

No obstante, hay también otra diferencia, tal vez más significativa, y es el papel primordial que desempeña el cúmulo de saber social, punto de arranque, y con el que interactúa el conocimiento tecno-organizativo. Benetton ilustra perfectamente lo que decimos. El denominado "modelo Benetton", ha sido un ejemplo de potenciación de sinergias de conocimiento e información con el entorno (mediante encuestas, marketing, etc.) que alimentan y retroalimentan a modo de "concurrent engineering" el circuito productivo... reproductivo -"integral"- de la mercancía; captura de lo que los "endógenos" consideran las "externalidades negativas" o "deseconomías". La investigación efectuada por Pantaleo (1996) ha destacado de qué modo un empresa aparentemente sin obreros ni fábricas ni redes comerciales, controlaba el trabajo, la circulación de dinero, la distribución, los flujos deseantes y el consumo. Un entramado que pivotaba alrededor de una marca: Benetton. Un emporio que logró involucrar a 250.000 trabajadores que "cooperaban" a través de un complejo reticular en que la producción estaba descentralizada y la red comercial era una externalización. Un complejo que, como ha remarcado aquel autor, se adentraba en la vida pública, exponía sus convicciones y emplazaba al consumidor a que se incorporara al "debate"; "la mercancía de Benetton invade el espacio público y contribuye así a la construcción del pensamiento de la opinión pública, dentro del nuevo rol de actor político" (Pantaleo, 1996: 174).

En fin, podemos afirmar que mediante la aplicación de los formatos postfordistas los flujos de trabajo, comportamiento y saber, dentro y fuera de la fábrica, adquieren una calidad y potencia inéditas. Unos formatos en los que el conocimiento se articula en torno a la nueva división del trabajo que borra la distinción entre el exterior y el interior de la fábrica. Además, la división del trabajo instaurada permite gestionar, en la interrelación con el entorno, inmediato o no, las necesidades de aporte de saber.

Otro aspecto en el que hay que hacer hincapié es en que las TIC no funcionan y se transforman de manera autónoma. En este sentido, el conocimiento del que se nutren las TIC, susceptible de convertirse en información digitalizada y/o en información sistémica, no brota espontáneamente; es, por el contrario, fruto de una complicada elaboración. En esta conversión el trabajo es justamente la actividad que se sitúa entre el saber bruto, conocimiento previo, social, materia prima, y la información. El trabajo se ubica entre la multitud de datos e informaciones y la forma adoptada por el conocimiento como saber 
operativo. Así, para Rullani (1998: 121), que llama "capitalismo cognitivo" a la forma de organización en que el valor económico se produce a partir del conocimiento, el conocimiento tácito y el conocimiento explícito que poseen los actores no se convierte de manera automática en mercancía. Son necesarias experimentaciones, pruebas, verificaciones, soluciones ad hoc que necesitan una aportación de trabajo. Tanto si se trata del aprendizaje práctico que precisa una instalación "lógico-racional" —comprobar un modelo- como de un proceso evolutivo — pruebas de ensayo/error para pasar un test/filtro- en un caso y otro, para que esto desemboque en conocimiento operativo, valorizante, se precisa trabajo. A este trabajo se le denomina "trabajo cognitivo".

Llegados a este punto, si partimos entre otros de las aportaciones de Nonaka\&Takeuchi (1995) y Konno (1998), podemos clasificar como sigue los tipos de conocimiento básicos:

a) Conocimiento incorporado sistemático, objetivado en las máquinas, tangible e intangible; abarca la tecnología y la organización.

b) Conocimiento desincorporado sistemático, implícito y explícito, que posee la fuerza de trabajo cualificada.

c) Saber desincorporado no sistemático, tácito y explícito; abarca desde una porción del know-how operario hasta el saber social difuso, que incluye el double-loop learning.

Es preciso señalar, no obstante, que el conocimiento sistemático, incorporado y desincorporado, sobre todo en lo tocante a la organización, antes que ser conocimiento funcional es, como se ha indicado, saber generado en las interacciones sociales y subjetivas. Nonaka y Konno han propuesto el modelo SECI como el instrumento que permite realizar el "ciclo de conversión", el paso de conocimiento tácito al explícito, lo cual requiere que el conocimiento tácito sea sustraído de su contexto originario.

Un aspecto relevante de estos procesos es, tal y como ha recalcado Shon Rethel (1977), la existencia de una homología entre la forma mercancía y la forma pensamiento, por el hecho de que la principal función del intelecto abstracto ha sido siempre la apropiación por una determinada clase social del saber elaborado por el trabajo manual de la otra.

Asimismo, cabe destacar la relación existente entre saber para la organización y "externalizaciones". En este punto es preciso subrayar el papel del saber difuso depositado en el TASG o en la subcontrata, una de cuyas exigencias radica en que en todo momento se pone a prueba la existencia o no de capacidad de respuesta rápida y eficaz, autoorganización en "tiempo real", que le permita desenvolverse en un entorno cambiante y no previsible. Nannicini $(2002,2005)$ ha mencionado estas cosas al indicar cómo el postfordismo ha captado el potencial de organización y autoorganizativo de la mujer en el trabajo doméstico para aplicarlo a la producción. La introducción de estas formas de organización del trabajo, que engloban el TASG, implican para el capital el desentenderse de unos costes y de unos requisitos que corresponden a unas cualificaciones complejas pero inmedibles, indispensables en un medio -"caos sistémico"- impredictible (Bologna, 2006: 91 ss.). 
En suma, podemos afirmar que con el postfordismo la fábrica deviene empresa socioterritorial -sociofactoría- que se despliega más que como productora de bienes y servicios, aunque también, como fábrica de comportamientos y "formas de vida" y, como un aspecto particular, como "fábrica de mentes" (Minsky, 1989). Pero, también, ocurre que en la inteligencia y en el saber colectivo, en el mind body process social, el capital halla su principal yacimiento de materia y energía para la valorización.

Esto significa, por tanto, que se delinea un bucle -"bucle sociocognitivo"- que se repite una y otra vez, y con el que la producción integral parasita el saber social y las "formas de vida" para convertirlos en "conocimiento valorizante" y, simultáneamente, dicho circuito produce "formas de vida" que lo retroalimentan. Se trata, a fin de cuentas, de una relación y de unos flujos circulares y autorreferenciales que se conforman con arreglo a una estructura jerárquica que obtiene su coherencia lógica y valorativa de cierta axiomática que es, en definitiva, un constructo.

\section{MÁQUINAS LINGÜISTICAS}

Un aspecto ineludible del postfordismo es el lenguaje y su función en los sistemas de comunicación. Sobre este particular, Marazzi $(2002,2003)$ ha enfatizado la importancia del lenguaje en el postfordimo y en la new economy. Se considera que en estos procesos el trabajador actúa como "máquina lingüística". Los sistemas de signos aparecen como un factor directamente productivo al quedar unidas producción y comunicación hasta el punto de que ésta se convierte en elemento potenciador de la economía. Sin embargo, no se trata de cualquier lenguaje, sino de un tipo particular de lenguaje, que es el que crea organización; literalmente: "A fin de ligar mejor la producción a las oscilaciones del mercado, el proceso de trabajo se estructura para fluidificar al máximo la circulación de la información gracias a la cual se puede responder en tiempo real a las exigencias del mercado. La comunicación de información hará uso, pues, de un lenguaje ágil, funcional a tal objetivo, de un lenguaje lógico-formal que permita, en el momento mismo en el que transmite información, suscitar actos de trabajo esenciales para la obtención del objetivo establecido" (Marazzi, 2003: 22). Dicho lenguaje tiene símbolos, signos y códigos abstractos que al ser compartidos permiten que la comunicación sea viable. El carácter lógico y formal de este lenguaje, con reglas de formación y transformación, hace que las acciones y los procesos funcionen como "máquinas lingüísticas" que se apoyan en "bancos de datos". Sin embargo, no es posible omitir que tomar en consideración solamente las expresiones traducibles al lenguaje binario excluye una variedad de signos y expresiones, que también son funcionalizados, que se rigen por otros criterios -pragmáticos, no siempre metódicos- y que son parte de las hablas y de la comunicación microsocial; una lectura factible si introducimos una visión del lenguaje en tanto que pragmática que engloba la "modalidad".

Así pues, lo que verificamos con el postfordismo es que la aprehensión de lenguajes $y$, en general, de saberes que pueden ser convertidos en información abarca, a modo 
de reducción de complejidad luhmanniana, una amplia variedad de expresiones y de conjuntos de signos.

Por otro lado, no hay que olvidar que una parte fundamental del lenguaje y de la comunicación es el contexto. Habida cuenta de que el contexto no es algo distinto y que rodea las cosas o las situaciones desde un supuesto afuera. Esta visión dualista no tiene en cuenta que el contexto es constitutivo de los propios procesos económicos y sociales (políticos, culturales, etc.). Podemos afirmar, por tanto, que el contexto no está ahí, al lado, adyacente, sino que es parte de lo mismo. El contexto se configura y es configurado mediante las interrelaciones sociales establecidas, lo que incluye, necesariamente, las dimensiones performativa y simbólica. Sobre este particular, Rullani (1998: 121 y ss.) distingue entre "conocimiento contextual" y "conocimiento codificado" o conocimiento que se prefigura como conocimiento que valoriza. En las sinergias entre ambos tipos de saber, en la relación con el entorno, el lenguaje resulta vital. Un lenguaje con un "código", entendiendo por tal una serie de convenciones que posibilitan la sustitución por otros significantes de los significantes de los signos preexistentes, habida cuenta de que aquellos significantes resultan más adecuados que estos (Martinet, 1988).

\section{HETERONOMÍA Y AUTONOMÍA}

Así las cosas, en este marco diverso, aunque las directrices estratégicas, las funciones de gobierno y determinados procesos básicos responden al modelo postfordista, ello no obsta para que, por lo que se refiere a la fuerza de trabajo, haya, como es sabido, amplias franjas neofordistas y neotayloristas de trabajadores, tanto en las áreas centrales como en la periferia capitalista, cuyas condiciones laborales remiten al Manchester de fines del XVIII y al colonialismo más vetusto. Un dato es de por sí suficientemente ilustrativo de la precariedad impuesta en el mundo del trabajo: según el Informe de la OIT del 2005 la mitad de los trabajadores del planeta perciben unas remuneraciones que se sitúan por debajo de los dos euros diarios. Por otra parte, estaría la proliferación del modelo contingent job o contratación fuera de reglas y la prolongación de la jornada de trabajo. No deja de ser significativo que de estas condiciones de precariedad no hayan podido escapar ni tan siquiera algunas secuencias postfordistas. Este hecho se debería a que, como advirtió Noble $(1984,2001)$, muchas veces en las decisiones sobre tecnología prevalecen motivos políticos, culturales y psicológicos, en lugar de razones técnicas y económicas como cabría esperar de unos procesos cuya lógica en principio debería corresponder a una teleología optimizadora en términos de racionalidad técnica y productiva.

En cualquier caso, lo que hemos podido constatar es que los sistemas organizativos y las técnicas de fabricación neo/postfordistas, no sólo no han ayudado a resolver las incertidumbres de un escenario turbulento como es el del periodo actual, sino que han contribuido a agudizar viejas contradicciones y a la aparición de otras nuevas. Éstas son, grosso modo, algunas de ellas: 
a) En los nuevos procesos tecno-organizativos se acrecienta la abstracción del trabajo, lo que repercute en la medida del valor. El grado de abstracción de algunos de los elementos básicos (cooperación, comunicación, modalidad,...) de la actividad laboral vinculada a las TIC y a la organización postfordista es muy superior al que se daba con el "modelo fordista". Hay que precisar aquí que en este plano del análisis no nos referimos a una actividad concreta, sino al trabajo genérico, social, promedio, que permite fijar el valor promedio de las mercancías, el cual dependía, en principio, hasta el fordismo, de las unidades en valor-tiempo que contenían las mercancías. Además, la pérdida de homogeneidad, imprevisibilidad y complejidad de los componentes del valor, particularmente en el knowledge worker y en el TASG, provoca fundamentalmente que aquél se autonomice del espacio y del tiempo. En efecto, con el postfordismo en muchos procesos el contenido de las operaciones agrupa un sinfín de componentes intangibles: imaginación, creatividad, reflexividad, eficacia comunicativa, capacidad de autorregulación en un clima de inestabilidad, habilidades relacionales, esquemas epistémicos y metodológicos, etc., cuyo denominador común estriba en que son competencias inconmensurables y no cuantificables. Al propio tiempo hay que tener en cuenta ciertos elementos del entorno, de lo que la ortodoxia categoriza como "deseconomías", en la medida en que son inseparables de las actividades que incorporan conocimiento a la producción. Una de las consecuencias de estos cambios es que se ponen en entredicho los instrumentos de contabilidad y seguimiento de la ley del valor. La causa más inmediata de esto reside en que aquellos instrumentos no admiten la imposibilidad de algoritmizar los ingredientes del conocimiento valorizante, de aquello que en realidad es condición indispensable del crecimiento y del aumento del valor del capital (Moulier, 2004), y las modificaciones que conlleva el trabajo postfordista. A partir de aquí, la ley de valor, como parte de una axiomática que se apoya en una relación de poder asimétrica que impone la medida del valor, pasa de ser ley económica a ser ley política; ley de poder, mando. Un ejemplo al respecto son los valores de las mercancías en lo regímenes monopolista y oligopolista a los que se hacen corresponder precios de monopolio y de oligopolio. La gestión de lo económico ha devenido, por tanto, administración política.

b) Otro elemento a destacar es que en la medida en que el conocimiento se prefigura como uno de los pilares de la producción integral, la privatización del saber en forma de patentes, marcas, contratos de usufructo, etc., se erige en obstáculo para el desarrollo del propio conocimiento. Por otra parte, la llamada eufemísticamente "economía del conocimiento" - la producción de valor a partir de las TIC y de la organización postfordista - convierten en obsoletos algunos postulados básicos de la tradición neoclásica, tal y como sucede con los presupuestos de la escasez y de los rendimientos decrecientes, ya que una economía cuya materia prima es el saber, es decir, algo que constituye un filón ilimitado, los rendimientos son crecientes y, además, el coste de su reproducción y circulación a través de las redes telemáticas tiende a cero, por lo que no se puede hablar de escasez, sino de abundancia, pero de abundancia en términos de mercado. 
c) Así pues, si el conocimiento aparece como una fuerza productiva primordial, pero, al propio tiempo, su desarrollo idóneo depende de la inexistencia de constricciones y de las sinergias establecidas -diversidad de formas de cooperación social que engloban al trabajo y al no trabajo- sucede que el principio de propiedad privada que regula la normativa del copyright, de los derechos de autor y de la patentes, al dificultar la libre circulación e intercambio de conocimientos, emerge como el principal impedimento para el desarrollo de aquella fuerza productiva. Sin duda cabe, esta contradicción está en el fondo de la crisis del e. business.

d) Por lo que se refiere a las contradicciones en la esfera psicosocial, podemos sacar a colación trastornos y patologías detectados en determinadas circunstancias en relación con el trabajo neo/postfordista y con el modus vivendi a él asociado. Aquí destaca por su particular relevancia la "disociación psíquica", configuración específica del mind body process; porción de las estructuras y procesos psicosociales y de la división social y técnica del trabajo, en concreto de lo que se categoriza como trabajo cognitivo; en este supuesto las funciones cognitivas y relacionales. En este supuesto, las funciones cognitivas y relacionales obligan al desdoblamiento del individuo. En efecto, se dibuja una línea invisible que depende de elementos materiales (dispositivos de poder y control tangibles, diseño de la red que depende de nodos y centros, etc.) e inmateriales (pautas, lenguaje, símbolos del poder) que separa al sujeto/objeto en tanto que actor virtualmente autónomo del sujeto/objeto convertido en simple objeto -sujeto sujetadoque actúa como apéndice mentalmente activo, partícipe, pero, a la vez, sometido a un nexo que se superpone, heterónomo. Estos nexos no son únicamente externos, impuestos desde fuera; tienen otra cara, interna, la de la asimilación, interiorización, de las formas de cooperación, de la sumisión. Sin embargo, ambas caras son inseparables, parte de los mismos procesos, porción de un conjunto que a través de las redes que se tejen, de la organización reticular, inextricable, que funciona en tanto que integrante de la "mente social cooperante". Mente sociocognitiva disociada en la que, como en el "pliegue epicúreo" de Foucault, no hay un adentro y un afuera, porque el afuera ya ocupa, coloniza, el adentro. La autonomía del individuo ha sido, por tanto, destruida, revertida hacia los fines de la valorización (vertiente económica) y la obediencia (vertiente políticocultural). Cabe mencionar aquí la afirmación de Gorz (1998) en la que indica, a diferencia de lo sostenido por Adam Smith, que el capital al organizar la producción e instaurar la división del trabajo perseguía no sólo la productividad, sino que también buscaba el control sobre la fuerza de trabajo. Tal estado de cosas está, por cierto, en la raíz de los trastornos y patologías ligados a las nuevas condiciones sociolaborales y de los cuadros clínicos en que aparece: estrés, pérdida de identidad, ansiedad, depresión, ausencia de sentido, etc., lo cual pone de manifiesto, en el fondo, que se ha producido una grave perdida de autonomía y de estabilidad psíquicas. Según Daubas y Thébaud (2002), en las condiciones de "trabajo flexible" (flexibilidad en el tiempo de trabajo y sin horario fijo) se detecta que las mujeres sufren estrés (51\%), dolor de cabeza (27\%) e insomnio $(22 \%)$; y los hombres estrés (39\%), irritabilidad (19\%) e insomnio (19\%). Asimismo, en 
Cataluña, el Institut Català de la Salut hizo público en 2007 que el 40\% del consumo de fármacos en esta comunidad autónoma correspondía a medicamentos ansiolíticos y antidepresivos.

e) Por fin, en este apartado abordaremos lo que bien puede ser considerado el núcleo duro de las contradicciones en el trabajo y en la tecno-organización, sobre todo en las secuencias postfordistas, a saber: la dicotomía provocada en los sujetos/objetos por unas estructuras y unas relaciones que potencian la autonomía y cooperación grupal de los sujetos/objetos y de los agregados, pero que, por otra parte, están diseñadas y evolucionan con arreglo a unos objetivos impuestos por la lógica de la valorización, producto de la existencia de un nexo jerárquico, disfrazado en ocasiones de horizontalidad, muy preciso. Se generan, por consiguiente, dinámicas que discurren en sentido opuesto unas con respecto a otras, a saber: las corporaciones promueven la cooperación mediante las implantación de sistemas co-implicativos, pero, a su vez, la "dualización" y el autoritarismo del cosmos laboral favorece la disgregación de lo social y de la individualidad con efectos tales como: desidentificación, impotencia, desamparo, retracción, soledad deambulatoria.

f) Por otra parte, si como se ha indicado, la reestructuración supuso la desarticulación de unos comportamientos y relaciones que discurrían en sentido contrario, o mejor antagonista, respecto del flujo del plusvalor, sin embargo, en una fase posterior, los sistemas de innovación reconvierten aquellas modalidades de cooperación antagonista y las transforman en modalidades sociocognitivas de cooperación social productiva. Podríamos decir que el capital se moderniza a través del conflicto y de la crisis. Éste es, por tanto, a fin de cuentas, ante todo por lo que se refiere a los sistemas organizativos, el significado del cambio técnico y organizativo: paso de la disciplina individualizada y fragmentaria fordista y tayloriana a la cooperación y el control multidisciplinar y social postfordista.

No obstante, llegados a este punto, a pesar de todo lo dicho hasta este momento, aun teniendo en cuenta que no pueda haber expansión real de la riqueza sin valorización, sin crecimiento del valor real tangible 0 intangible, y en este punto hay que decir que juega un papel crucial el postfordismo, es indispensable efectuar una puntualización. En sentido sentido, lo que se constata es que los cambios operados por lo que se refiere a las relaciones de poder político y económico en las últimas cuatro décadas han conducido a que la lógica financiera tuviera un papel decisivo en la esfera económica; mayor peso específico de la lógica del activo -asset paradigm- respecto de la lógica productiva, que se guía por el plusvalor, que hace que sean los "activos financieros" y no la actividad productiva lo que finalmente determine, en la etapa actual, la deriva económica y, por tanto, los procesos de trabajo y tecno-organizativos. Ello quiere decir que se da, si partimos del binomio economía productiva/economía financiera como espacios relativamente autónomos (múltiples conexiones: créditos, préstamos, titulizaciones, etc.), una preponderancia de la segunda sobre la primera. (Aglietta, 1998, 
2001; Brenner, 2003; Harvey, 2006, 2007; Marazzi, 1998, 2002 ; Palazuelos, 1998; Wallerstein, 2005) Esta situación insólita, que se gesta en 1971, con el fin de la paridad dólar-oro y que, por consiguiente, supuso liquidar uno de los pilares de los acuerdos de Bretton Woods, inauguró unas décadas de glorificación de la "mano invisible" que, de hecho, han atravesado fases sumamente críticas. Sobre este particular, no deja de tener interés el análisis esbozado por Samir Amin (2008), a raíz de la "crisis financiera" (la crisis es estructural), en el que indica que el centro del poder económico se ha desplazado de la producción de plusvalía en la producción a la redistribución de los beneficios generados por las inversiones financieras. Desde esta óptica, ya no estaríamos fundamentalmente en presencia de una economía de mercado, sino de un "capitalismo de oligopolios financiarizados"; o bien, de un capitalismo rentista.

Antes de terminar, llegados a este punto, voy a retomar la noción de escenario heterogéneo, híbrido, para aludir a lo que constituye uno de los dispositivos neurálgicos, clave de bóveda del postfordismo, el "bucle sociocognitivo", según el cual los flujos derivados de la producción-reproducción-distribución conforman la subjetividad social en el territorio y, al mismo tiempo, a través de múltiples interacciones, la subjetividad social retroalimenta los flujos de producción. Aunque, ese carácter híbrido que le atribuimos no impide que desde este punto de vista se considere que el paso del fordismo al postfordismo haya significado, a resultas de la reestructuración que tuvo lugar entre los años 70 y 80 , una verdadera mutación, no un mero ajuste, o una reforma continuista respecto de lo ya existente. Pero una mutación enmarcada dentro del nexo de poder que es el capital. Aduciremos de manera esquemática varios argumentos al respecto, a saber:

a) Relevancia de las formas organizativas y distintos cambios que incluyen la adopción descentralizada del formato supply chain.

b) Heterogeneidad y precariedad de las condiciones laborales de la fuerza de trabajo.

c) Implantación de las TIC.

d) Y, sobre todo, captura y fagocitación el ámbito territorial, dentro y fuera de la fábrica, del saber social convertido en conocimiento valorizante desde la división del trabajo.

Ciertamente, las consecuencias que se desprenden de la aplicación de estos nuevos sistemas tecno-organizativos incidirán de manera decisiva en el trabajo y en las condiciones de vida (Reich, 1992; Aronowitz\&Di Fazio, 1994; Rifkin, 1997; Gorz, 1998; Berardi, 2001; Quintana, 2005). Sobre este particular, parece claro que las TIC y el new management permiten disponer de un gigantesco potencial de creación de riqueza en el ámbito de la economía productiva y, paralelamente, reducir la demanda de fuerza de trabajo y de tiempo de trabajo. Ahora bien, si tenemos en cuenta el background teórico (monetaristas, teorías del equilibrio, etc.) en que se apoyan y el contexto socioeconómico y político, lo que han originado este conjunto de modificaciones es un aumento de la 
exclusión, pero no de la calidad de vida (Arrighi, 2007). Sin embargo, por otro lado, desde cierta perspectiva, de algún modo condicionada por el trasfondo de la monetarización de las relaciones sociales y de la expansión de la racionalidad financiarizadora, se torna más evidente la opción zero work (Berardi, 1994; 2001).

Finalmente, quiero destacar, a modo de contrapunto, en este escenario paradójico de los contextos postfordistas, en el se impone una relación disociativa de cooperación lexclusión, la oposición que existe entre, por una parte, el trabajo como actividad de naturaleza heterodeterminada en cualquiera de sus formatos tecno-organizativos $y$, por otra parte, los tramos discontinuos, praxis desmercantilizada y contraria al poder heterónomo que cabe conceptuar como "actividad colectiva autodeterminada". Este inciso me permite, por fin, asimismo, evocar un interesante y oportuno fragmento de Lewis Mumford (1971), crítico riguroso de la técnica, en el que pone de relieve su profundo rechazo a "las abstracciones del constante progreso tecnológico y de la acumulación de dinero" (p. 284).

\section{Referencias Bibliográficas}

Aglietta, M. (2001), Macroéconomie financièr. 1. Finance, croissance et cycles, París, La Decouverte.

Aglietta, M. y A. Orléan (1998), La monnaiesouveraine, París, Odile Jacob.

Aglietta, M. (2001), Macroéconomie financière. 2 Crisis financière et régulation monétaire, París, La Decouverte.

Amatori, F. et al. (1997), Storia del capitalismo italiano dal dopoguerra ad oggi Roma, Donzelli.

Amin, S. (2008), www.michelcollon.info

Aronowitz, S. y W. Di Fazio (1994), The Jobless Future, Minneapolis, University of Minnesota.

Arrighi, G. (2007), Adam Smith in Beijing. Lineages of the Twenty-first Century, Londres, Verso.

Arrighi, G. y J. S. Beverly (2001), Caos y orden en el sistema mundo, Madrid, Akal.

Bagnasco, A. (2003), Società fuori squadra, Bolonia, II Mulino.

Berardi, F. (1994), Lavoro Zero, Roma, Castelvecchi.

(2001), La fabbrica dell'infellicità, New Economy e movimento del cognitariato, Roma, Derive Approdi.

Bologna, S. y A. Fumagalli (coord.) (1997), Il lavoro autonomo di seconda generazione, Milano, Feltrinelli.

Boltanski, L. y E. Chiapello (2002), El nuevo espiritu del capitalismo, Madrid, Akal.

Bourdieu, P. (2000), Les structures sociales de l'economie, París, Seuil. 
Brenner, N. y N. Theodore (coord.) (2002), Spaces of Neoliberalism, Oxford, Blackwell Publishing.

Brenner, R. (2003), La expansión económica y la burbuja bursátil, Madrid, Akal.

Castells, M. y P. Hall (1994), Las tecnópolis del mundo, Madrid, Alianza Editorial.

Cillario, L. (1996), L'economia degli spettri, Roma, Manifestolibri.

Daubas, V. y A. Thébaud (2002), Organisation du travail et santé dans l'Union Européenne, Fondation européenne pour l'améliorament des conditions de vie et de travail. Office des publications des officielles de Communautés Européenes.

Delaunay, J. C. (coord.) (2001), Le capitalismo contemporaine. Questions de fond, Paris, L'Harmattan.

Deleuze, G., F. Guattari (1988), Mil mesetas, Valencia, Pre-Textos.

European Innovation Scoreboard, 2004, Comparative Analysis of Innovation Performance. European Comisión.

Fadini, U. y A. Zanini (2001), Lessico postfordista, Milano, Interpone.

Gaudemar, J. P. (1981), La movilización general, Madrid, La Piqueta.

Gorz, A. (1998), Miserias del presente, riqueza de lo posible, Buenos Aires, Paidós.

(2003), L'immateriel. Conaissance, valeur et capital, Paris, Galilée.

Harvey, D. (2004), El nuevo imperialismo, Madrid, Akal.

(2006), Spaces of Global Capitalism. Towards a Theory of Uneven Geographical Development, Londres, Verso.

(2007), Breve historia del neoliberalismo, Madrid, Akal.

Herrera, R. y C. Vercellone (2003), Sommes- nous sortis du capitalisme industriel?, Paris, La Dispute.

Ilardi, M. (1999), Negli spazi vuoti della metrópoli, Torino, Bollati Boringhieri.

Instituto per la Promozione Industriale (2003), L'esperienza italiana dei distritti industriali, Roma, Ministero delle Attività Produttive.

Kirby, P. (2002), The Celtic Tiger in Distress Growth with Inequality in Ireland, Londres, Palgrave.

Marazzi, C. (1998), E il denaro va, Torino, Bollati, Boringhieri.

(2002), Capitale\&linguaggio, Roma, Derive Approdi.

(2003), El sitio de los calcetines, Madrid, Akal.

Martinet, A. (1988), Sintassi generale, Bari, Laterza. 
Minsky, M. (2001), La società della mente, Milano, Adelphi Edizioni.

Moulier, Y. et al. (2004), Capitalismo cognitivo, Madrid, Traficantes de sueños.

Mumford, L. (1971), Technique and Human Developmen, Orlando, Harcourt Press.

Nannicini, A. et al. (2002), Le parole per farlo, Roma, Derive Approdi.

(2005), Ponencia para Jornada de debate «La f@brica i la societat», Barcelona, MACBA.

Navarro, V. (2006), El subdesarrollo social en España, Barcelona, Anagrama.

Negri, A. (1979), Marx oltre Marx, Milano, Feltrinelli.

(1992), Fin de siglo, Barcelona, Paidós/ICE-UAB.

Noble, D. (1984), Forces of Production. A Social History o Industrial Automation, Oxford, Oxford University Press.

(2001), La locura de la automatización, Barcelona, Alikornio.

Nolan, B. et. al. (2002), Bust to Boom? The Irish Experience of Growth and Inequality, Dublin, Institute of Public Administration.

Nonaka, A y H. Takeuchi (1995), The Knowledge Creating Company, New York, Oxford University Press.

Palazuelos, E. (1998), La globalización financiera, Madrid, Síntesis.

Pantaleo, F. (1996), "Agencements spatiaux, la séduction du marché", Futur Antérieur, 35-36 : 157-176.

Piore, M. J. y Ch. F. Sabel (1990), La segunda ruptura industria, Madrid, Alianza Editorial.

Quintana, F. y N. Aut (2002), Asalto a la fábrica, Barcelona, Alikornio.

Quintana, F. (2005), "Trabajo, no trabajo y neo/postfordismo", Cadernos de Psicología Social do Trabalho, 8: 43-58.

(2006), "Metrópoli y subsunción múltiple", Cadernos de Psicología Social do Trabalho. 9: 15-40.

Rhodes, M. y Y. Meny (1998), The Future of Welfare, Palgrave, Mc. Millan.

Rullani, E. et al. (1998), Capitalismo e conoscenza, Roma, Manifestolibri.

Vincent, J.M. (1998), "Sortir du travail", Futur Antérieur, 43 : 85-97.

Virno, P. (2001), Grammatica della moltitudine, Catanzaro, Rubbettino.

Wallerstein, I. (2005), La crisis estructural del capitalismo, México, Contrahistorias.

(2007), Geopolítica y geocultura: ensayos sobre el moderno sistema mundial, Barcelona, Kairós. 
Zanini, A. y U. Fadini (2001), Lessico postfordista, Milano, Interpone.

Zarifian, P. (1996), Travail et communication, París, PUF.

RECIBIDO: $09 / 06 / 06$

ACEPTADO: 26/06/08 